\title{
On the Statistical Estimation of Diffusion Processes: A Partial Survey*
}

\author{
Rubens Penha Cysne ${ }^{* *}$
}

\begin{abstract}
Data available on continuous-time diffusions are always sampled discretely in time. In most cases, the likelihood function of the observations is not directly computable. This survey covers a sample of the statistical methods that have been developed to solve this problem. We concentrate on some recent contributions to the literature based on three different approaches to the problem: an improvement of the Euler-Maruyama discretization scheme, the employment of Martingale Estimating Functions, and the application of Generalized Method of Moments (GMM).
\end{abstract}

Keywords: Diffusion, Continuous-time, Estimation.

JEL Code: C13, C22, C32, G0.

\footnotetext{
* Submitted in April 2004. Revised in October 2004.

** Professor at the Graduate School of Economics (EPGE/FGV) of the Getulio Vargas Foundation and, in 2004, a Visiting Scholar at the Department of Economics of the University of Chicago. E-mail: rpcysne@uchicago.edu.
} 


\section{Introduction}

A large number of models in economics and finance describe the time evolution of dynamic phenomena in a continuous-time stochastic framework. Interest-rate models, for instance, are nowadays frequently formulated in terms of nonlinear stochastic differential equations. This implies the need to estimate the parameters of such models. However, in practice, the data used for such inference is always of a discrete nature, sampled at discrete intervals of time. This leads us to a very specific statistical problem, that has been a subject of active academic research for many years, dating back to the seminal work of Phillips (1959).

The aim of this paper is to provide a partial survey of some of the techniques used in the statistical inference of diffusions. The qualification "partial" here is used to alert the reader that we review only a fraction of the many techniques that have been devised to deal with the problem: an improvement of the EulerMaruyama discretization scheme, the employment of Martingale Estimating Functions, and the application of the Generalized Method of Moments (GMM).

The Euler-Maruyama approach employs a discrete-time approximation to the continuous system. The estimation of the discrete-time model is then accomplished by maximum likelihood. Martingale Estimating Functions, the second technique studied here, and whose prime example is the score function (the gradient of the likelihood function with respect to the parameters of interest), represent a particular type of estimating functions, distinguished by the nice property of allowing the use of all the available machinery of Martingale Theory. The utilization of this machinery is particularly helpful in the derivation of large-sample properties of the estimators, in which case the Martingale Central Limit Theorem (Billingsley, 1961) can be used.

Finally, a GMM estimator (Hansen, 1982) is a vector that minimizes a distance function, properly defined, of the sample moments from zero. Approximation results that justify the use of GMM in the estimation of diffusion processes are found in Hansen and Scheinkman (1995).

Rigorous definitions of a diffusion process can be found in Krylov (1980) or in Karatzas and Shreve (1991). Loosely speaking, a diffusion process is a Markov process with continuous sample paths which can be characterized by an infinitesimal generator (which we are going to define below). The simplest diffusion process is the Wiener process, the stochastic process that corresponds to the Brownian Motion.

As a general point of departure for the type of problem in which we shall be interested, consider the stochastic integral equation relative to a stochastic process $X_{t}$ in $\mathbb{R}^{d}$ :

$$
X_{t}=X_{0}+\int_{0}^{t} h\left(\theta, s, X_{s}\right) d s+\int_{0}^{t} \sigma\left(s, X_{s}\right) d W_{s}, \quad 0 \leq s \leq t
$$

$\theta \in \mathbb{R}^{k}$ denoting parameters of the model (we are considering that $\sigma$ does not 
depend on $\theta$ ). In this equation, $X_{0}$ is an $\mathcal{F}_{0}$-measurable function independent of $\left\{W_{u}-W_{v}, u \geq v \geq 0\right\}$ and $W$ is a standard Brownian Motion.

Let $\mathcal{F}_{0, t}^{W}$ be the completion of the $\sigma$-algebra generated by $\left\{W_{u}, t \geq u \geq 0\right\}$. Denote by $\mathcal{F}_{0, t}$ the $\sigma$-algebra generated by $\mathcal{F}_{0}$ and $\mathcal{F}_{0, t}^{W}$. To simplify notation, make $\mathcal{F}_{0, t}=\mathcal{F}_{t}$. Now suppose $h: \mathbb{R}^{k} \times \mathbb{R}_{+} \times \mathbb{R}^{d} \rightarrow \mathbb{R}^{d}$ is $\mathcal{F}_{t}$-measurable and $\sigma$ is a $(d \times m) \mathcal{F}_{t}$-measurable matrix with $\sigma_{i j}: \mathbb{R}_{+} \times \mathbb{R}^{d} \rightarrow \mathbb{R}$. Under a set of Lipschitz conditions (see, e.g., Prakasa (1999) or Oksendal (2000), subsection 5.2), the equation:

$$
d X_{t}=h\left(\theta, t, X_{t}\right) d t+o\left(t, X_{t}\right) d W_{t}, \quad X_{0}=x_{0} \in \mathbb{R}^{d}, t \geq 0
$$

has a unique and continuous solution $X_{t}(t, \omega)$ and, for each $t \geq 0, X_{t}$ is $\left(\mathcal{F}_{t}\right.$ Borel)-measurable. $\left\{X_{t}\right\}$ is a continuous Markov process relative to $\mathcal{F}_{t}$.

The process is called homogenous if $h\left(\theta, s, X_{s}\right)=h\left(\theta, X_{s}\right)$ and $\sigma\left(s, X_{s}\right)=$ $\sigma\left(X_{s}\right)$. In general, we shall be interested in homogenous processes in $\mathbb{R}(d=1)$.

For the purpose of stochastic modelling, we can think of a diffusion process as a continuous version of a process:

$$
X_{t+1}=f\left(X_{t}, s_{t}\right)+\epsilon_{t}
$$

where $X_{t}$ stands for the state at the $\mathrm{t}^{\text {th }}$ generation, $s_{t}$ for a random or fixed parameter at the $t^{t h}$ generation and $\epsilon_{t}$ for a noise.

Example 1 (Wiener Process with Drift $\mu$ and Diffusion $\sigma^{2}$ ) :

$$
d X_{t}=\mu d t+o d W_{t}, \quad X_{0}=0 \in \mathbb{R}, t \geq 0
$$

In this case $X(t)-X(s), t>s>0$, is normal with independent increments, mean $E(X(t)-X(s))=\mu|s-t|$ and $\operatorname{Var}(X(t)-X(s))=o^{2}|s-t|$.

Ideally, parametric inference for diffusion processes should be based on the likelihood function. Since such processes are Markovian, the likelihood function (given that the initial point is known) is the product of transition densities. However, the transition densities $f_{k}$, on which the maximum likelihood function has to rely, can be obtained in closed-form only in very specific cases. Given this hindrance to the direct application of the likelihood method, different alternatives have been proposed in the literature.

Pedersen (1995a,b) derived the estimators departing from approximations of the continuous-time likelihood function using simulation methods. More recently, Ait-Sahalia (2002) has proposed the use of closed-form approximations of the (unknown) likelihood functions based on Hermite polynomials. The estimator so obtained is shown by the author to converge to the true maximum likelihood estimator and to share its asymptotic properties.

The method proposed by Ait-Sahalia starts by making a transformation of the original process, from $X$ to $Z$. $Z$ is a process for which the Hermite expansion 
of the transition densities converges. It is the appropriate transformation of $X$, whose expansion starts with a $N(0,1)$ term. Since $Z$ is a known transformation of $\mathrm{X}$, the expansion of the density of $\mathrm{X}$ can then be obtained by the use of the Jacobian formula, thereby leading (analytically) to closed-form approximations of the maximum likelihood function.

As pointed out before, the first two techniques on which we shall concentrate here, the Euler-Maruyama discretization and the Martingale Estimating Functions, are based on replacements of the true likelihood function (which is not known) by some approximation. Such procedures, sometimes classified as indirect inference (Gourieroux and Jasiak, 2001), are usually followed by additional steps involving simulation and calibration (in order to improve the quality of the estimators). The third method object of our analysis will be the Generalized Method of Moments (GMM).

\section{Two Basic Applications in Finance}

Diffusion processes provide an alternative to the discrete-time stochastic processes traditionally used in time series analysis. The need of modelling and estimating such processes has been particularly important in finance and economics, where they are fitted to time series of, for instance, stock prices, interest rates, and exchange rates, in order to price derivative assets.

The applications shown below fall into a category (see subsection 4.2.1) in which the diffusion process is of a type such that the transition functions are known. In this case the parameters can be directly estimated by maximum likelihood. The reason we introduce such applications here is that they are a point of departure for more complicated models, which use other underlying diffusion processes, and which do not lead to transition functions that are known.

For instance, there is considerable evidence that the increments of the logarithm of the price of the stock used to price options in the Black and Scholes model are neither independent nor Gaussian, as implied by equation (2) below. This leads to the necessity of more complex estimation processes, the analysis of which is the purpose of this survey.

- Black and Scholes (1973). We present here the version of Campbell et al. (1997). Suppose we want to find the price $G(P(t), \tau)$ at time $t$, of an (European) option with strike price $X$ and expiration date $T>t$, with $\tau=T-t$. We assume that the relative changes of prices follow the equation:

$$
\frac{d P(t)}{d t}=k(t) P(t), P_{0} \text { given }
$$

with $k(t)=\mu+\sigma Z(t), Z(t)$ a white noise and $\mu$ and $\sigma$ constants. In Itô's representation: 


$$
d P(t)=\mu P(t) d t+\sigma P(t) d W(t), \quad t \geq 0
$$

$W(t)$ standing for a standard Brownian motion. The hypothesis of the model is that $P(t)$ describes the stock price upon which the option price is based. Now suppose (we omit the arguments of the function $P($.$) ) that an initial investment I$ is allocated in options and stocks according to

$$
I=G(P, t)+\alpha P
$$

Using Itô's Lemma:

$$
\begin{aligned}
d G(P, t) & =d t\left[\mu P G_{P}+G_{t}+\frac{1}{2} P^{2} \sigma^{2} G_{P P}\right]+P G_{P} \sigma d W \\
d I(t) & =d t\left[\left(\alpha+G_{P}\right) P \mu+G_{t}+\frac{1}{2} P^{2} \sigma^{2} G_{P P}\right]+\left(\alpha+G_{P}\right) P \sigma d W
\end{aligned}
$$

The risk is zero when $d I(t)$ does not depend on the stochastic component $\left(\alpha+G_{P}\right) P \sigma d W$, which implies $\alpha+G_{P}=0$. In this case the expected income per unit of time is $G_{t}+\frac{1}{2} P^{2} \sigma^{2} G_{P P}$. Denoting by $r$ the risk-free rate, the no-arbitrage condition demands:

$$
G_{t}+\frac{1}{2} P^{2} \sigma^{2} G_{P P}=r I
$$

Using (3) and the no-risk condition once more:

$$
G_{t}+\frac{1}{2} P^{2} \sigma^{2} G_{P P}=r(G+\alpha P)=r\left(G-G_{p} P\right)
$$

from which we get:

$$
G_{t}+\frac{1}{2} P^{2} \sigma^{2} G_{P P}-r\left(G-G_{p} P\right)=0
$$

Since the (European) option is only exercised if the price at time $\mathrm{T}$ is no less than the strike price $\mathrm{X}$ :

$$
G(P(T), T)=\max (0, P(T)-X)
$$

Solving (4) with condition (5) gives the price of the option as a function of time and of the parameter (which must be estimated) $\sigma$.

- Cox et al. (1985): ${ }^{1}$ In this model the state variable follows a diffusion process given by:

$$
d X_{t}=\left(\alpha+\theta X_{t}\right) d t+\sigma \sqrt{X_{t}} d W_{t}
$$

${ }^{1}$ This process is usually abbreviated by CIR-SR, with SR denoting square root (because of the $\sqrt{X_{t}}$ term). One usually denotes by CIR-VR the process, used in another work of these authors, in which the exponent of $X_{t}$ is $3 / 2$, rather than $1 / 2$. We shall come back to this process later in this text. 
In this case the parameters $\alpha, \theta$ and $\sigma$ are the purpose of the statistical estimation.

\section{The Generator of a Diffusion Process}

Let $f($.$) be a bounded twice continuously differentiable function, with bounded$ derivatives, and $X_{t}$ a generic time-homogeneous diffusion process defined on the probability space $(\Omega, \mathcal{F}, P)$. Let $\mathcal{Q}$ be the probability measure induced by $\mathrm{X}_{t}$ on $R^{n}$ (for any t) and $L^{2}(\mathcal{Q})$ be the space of Borel measurable functions $f\left(X_{t}\right): R^{n} \rightarrow R$, $\mathcal{Q}$-square integrable. In this space (not distinguishing between the space itself and the equivalent-classes space) we define, for $t \geq 0$, the family of operators:

$$
\Gamma_{t} f\left(x_{0}\right)=E\left[f\left(X_{t}\right) \mid X_{0}=x_{0}\right]\left(\equiv E^{0}\left(f\left(X_{t}\right)\right)\right.
$$

It can be shown that these operators $\left(L^{2}(\mathcal{Q}) \rightarrow L^{2}(\mathcal{Q})\right)$ are well defined $\left(f=f^{*}\right.$ $\mathcal{Q}$-a.e. $\rightarrow \Gamma_{t}(f)=\Gamma_{t}\left(f^{*}\right) \mathcal{Q}$-a.e. and $\left.\Gamma_{t}\left(f^{*}\right) \in L^{2}(\mathcal{Q})\right)$, a weak contraction $\left(\left\|\Gamma_{t}(f)\right\|\right.$ $\leq\|f\|$ ) and a semi-group (by the law os iterated expectations, $E^{0}\left(X_{t+s}\right)=$ $E^{0}\left(E^{t}\left(X_{t+s}\right)\right)$, implying $\left.\Gamma_{t+s}=\Gamma_{t} \Gamma_{s}\right)$.

In the remaining of this text, we will some times refer to the infinitesimal generator of a diffusion process $f\left(X_{t}\right)$. The infinitesimal generator gives a measure of the infinitesimal drift of a diffusion. For some functions $f \in L^{2}(\mathcal{Q})$ for which the limit below exists ( call it $\Psi$, a proper subset of $L^{2}(\mathcal{Q})$ ), this is defined as:

$$
\Lambda f\left(x_{0}\right)=\lim _{t \downarrow 0} \frac{\Gamma_{t} f\left(x_{0}\right)-f\left(x_{0}\right)}{t}, \quad t \geq 0
$$

$\Gamma$ and $\Lambda$ commute on $\Psi$ and $\Psi$ is dense in $L^{2}(\mathcal{Q})$.

We need a Proposition about the way how this generator materializes in the case of a particular diffusion process. The initial part of the proof is done in Hansen and Scheinkman (1995).

Proposition 1 Consider the one-dimensional diffusion process defined as solution to the stochastic differential equation:

$$
d X_{t}=b\left(X_{t} ; \theta\right) d t+\sigma\left(X_{t} ; \theta\right) d W_{t}
$$

where $W_{t}$ is a Wiener process. Let $L_{\theta}$ be a (differential) operator defined by:

$$
L_{\theta}=b(x ; \theta) \frac{d}{d x}+\frac{1}{2} \sigma^{2}(x ; \theta) \frac{d^{2}}{d x^{2}}
$$

Then $\Lambda f\left(x_{0}\right)=L_{\theta} f\left(x_{0}\right)$.

Proof We divide the Proof in six parts. 
$I$ - Write $Y_{t}=f\left(X_{t}\right)$, use (8) and apply Itô's formula to get:

$$
d Y_{t}=f^{\prime}\left(X_{t}\right)\left(b\left(X_{t} ; \theta\right) d t+\sigma\left(X_{t} ; \theta\right) d W_{t}\right)+\frac{1}{2} f^{\prime \prime}\left(X_{t}\right) \sigma^{2}\left(X_{t} ; \theta\right)\left(d W_{t}\right)^{2}
$$

II - Substitute $d t$ for $\left(d W_{t}\right)^{2}$ and integrate to get:

$$
\begin{aligned}
Y_{t}= & f\left(X_{t}\right)=f\left(x_{0}\right)+\int_{0}^{t}\left[b\left(X_{s} ; \theta\right) f^{\prime}\left(X_{s}\right)+\frac{1}{2} f^{\prime \prime}\left(X_{s}\right) \sigma^{2}\left(X_{s} ; \theta\right)\right] d s+ \\
& +\int_{0}^{t} f^{\prime}\left(X_{s}\right) \sigma\left(X_{s} ; \theta\right) d W_{s}
\end{aligned}
$$

III - By the construction of the Itô's Integral, for $u<t$,

$$
E^{u} \int_{0}^{t} f^{\prime}\left(X_{s}\right) \sigma\left(X_{s} ; \theta\right) d W_{s}=\int_{0}^{u} f^{\prime}\left(X_{s}\right) \sigma\left(X_{s} ; \theta\right) d W_{s}
$$

Using the definition of $L_{\theta}$ established by (9),

$$
Y_{t}-f\left(x_{0}\right)-\int_{0}^{t} L_{\theta} f\left(X_{s}\right) d s
$$

is a continuous martingale.

IV - Taking expectations conditional on $x_{0}$ :

$$
E\left[f\left(X_{t}\right) \mid x_{0}\right]-f\left(x_{0}\right)=E\left[\int_{0}^{t} L_{\theta} f\left(X_{s}\right) d s \mid x_{0}\right]
$$

$V$ - Using Frbini's theorem (by assumption, the integrand is quasi-integrable w.r.t the product measure) and definition (6):

$$
\frac{I_{t}^{\prime} f\left(x_{0}\right)-f\left(x_{0}\right)}{t}=(1 / t) \int_{0}^{t} \Gamma_{s}^{\prime} L_{\theta} f\left(x_{0}\right) d s
$$

$V I$ - Now take limits with $t \rightarrow 0$ on both sides of the above equation. The left side, by definition, is equal to $\Lambda f$. Therefore, the demonstration will be finished once we show that the limit of the right side equals $L_{\theta} f$. We need to show:

$$
\int_{R^{n}}\left\{\left[(1 / t) \int_{0}^{t}\left(\Gamma_{s} L_{\theta} f\left(x_{0}\right)-L_{\theta} f\left(x_{0}\right)\right) d s\right]^{2}\right\} d \mathcal{Q} \rightarrow 0
$$

Using the Cauchy-Schwarz (Hölder) inequality:

$$
\begin{aligned}
& \int_{R^{n}}\left\{\left[(1 / t) \int_{0}^{t}\left(\Gamma_{s} L_{\theta} f\left(x_{0}\right)-L_{\theta} f\left(x_{0}\right)\right) d s\right]^{2}\right\} d \mathcal{Q} \\
\leq & (1 / t)^{2} \int_{R^{n}}\left\{\int_{0}^{t}\left[\Gamma_{s} L_{\theta} f\left(x_{0}\right)-L_{\theta} f\left(x_{0}\right)\right]^{2} d s \int_{0}^{t} 1^{2} d s\right\} d \mathcal{Q} \\
= & (1 / t) \int_{R^{n}}\left\{\int_{0}^{t}\left[\Gamma_{s} L_{\theta} f\left(x_{0}\right)-L_{\theta} f\left(x_{0}\right)\right]^{2} d s\right\} d \mathcal{Q}
\end{aligned}
$$


Using Frubini again,

$$
\begin{aligned}
& (1 / t) \int_{R^{n}}\left\{\int_{0}^{t}\left[\Gamma_{s} L_{\theta} f\left(x_{0}\right)-L_{\theta} f\left(x_{0}\right)\right]^{2} d s\right\} d \mathcal{Q} \\
= & (1 / t) \int_{0}^{t}\left\{\int_{R^{n}}\left[\Gamma_{s} L_{\theta} f\left(x_{0}\right)-L_{\theta} f\left(x_{0}\right)\right]^{2} d \mathcal{Q}\right\} d s \\
= & (1 / t) \int_{0}^{t}\left\|\Gamma_{s} L_{\theta} f\left(x_{0}\right)-L_{\theta} f\left(x_{0}\right)\right\|^{2} d s
\end{aligned}
$$

which goes to zero by the assumption that $X_{t}$ is (Borel) measurable with respect to the product sigma-algebra (the sigma algebra generated by the measurable rectangles $A x B, A \in \mathcal{R}, B \in \mathcal{F}$ (this implies ${ }^{2}$ that, for each $\phi,\left\{\Gamma_{t} \phi, t \geq 0\right\}$ converges to $\phi$ as $t \downarrow 0)$ ).

\section{Maximum Likelihood Estimation (MLE)}

\subsection{Continuously observed data}

Likelihood methods for continuously observed diffusions are standard in the literature. We concentrate our exposition here on Prakasa (1999) and Basawa and Prakasa (1980). The important point to note is that likelihood function in this case can be obtained by a classical result on change of measure.

Consider the diffusion process (1). Note that the function $\sigma\left(s, X_{t}\right)$ does not depend on the parameter $\theta$. We assume that $\sigma\left(s, X_{t}\right)$ is known or, alternatively, that it is a constant, in which case it can be estimated from a standard quadraticvariation property of the Wiener process. Stokey (2000:2), presents this as an exercise.

Proposition 2 If $X$ is a $\left(\mu, \sigma^{2}\right)$ Brownian motion, then over any finite interval $[S, S+T]$ :

$$
Q V_{n} \equiv \sum_{j=1}^{2^{n}}\left[X\left(\frac{j T}{2^{n}}, \omega\right)-X\left(\frac{(j-1) T}{2^{n}}, \omega\right)\right]^{2} \rightarrow \sigma^{2} T, P-\text { a.e. as } n \rightarrow
$$

(QV stands for quadratic variation).

Proof We omit the $\omega$. Make

$$
\Delta_{j, n}=X\left(\frac{j T}{2^{n}}\right)-X\left(\frac{(j-1) T}{2^{n}}\right)
$$

Then $E\left(\Delta_{j, n}\right)=0$ and

$$
E\left(\Delta_{j, n}\right)^{2}=E\left(\frac{X\left(\frac{j T}{2^{n}}\right)-X\left(\frac{(j-1) T}{2^{n}}\right)}{\sqrt{\frac{T}{2^{n}} \sigma^{2}}}\right)^{2} \frac{\sigma^{2} T}{2^{n}}=\frac{\sigma^{2} T}{2^{n}}
$$

\footnotetext{
${ }^{2}$ See footnote 4 in Hansen and Scheinkman (1995).
} 
because $\frac{X\left(\frac{j T}{\left.2^{2}\right)-X\left(\frac{(j-1) T}{2{ }^{2}}\right)}\right.}{\sqrt{\frac{T}{2^{n} \sigma^{2}}}}$ is a $N(0,1)$ r.v. Define $x_{n}=\sum_{j=1}^{2^{n}}\left(\Delta_{j, n}\right)^{2}$. Then

$$
E x_{n} \equiv E\left(\sum_{j=1}^{2^{n}}\left(\Delta_{j, n}\right)^{2}\right)=\sigma^{2} T
$$

and

$$
E\left(x_{n}-\sigma^{2} T\right)^{2}=\operatorname{Var}\left(x_{n}\right)=\operatorname{Var}\left(\sum_{j=1}^{2^{n}}\left(\Delta_{j, n}\right)^{2}\right)
$$

By the independence of increments:

$$
\begin{aligned}
E\left(x_{n}-\sigma^{2} T\right)^{2} & =\sum_{j=1}^{2^{n}} \operatorname{Var}\left(\Delta_{j, n}\right)^{2}=\sum_{j=1}^{2^{n}} \frac{\sigma^{4} T^{2}}{2^{2 n}} \operatorname{Var}\left(\left(\frac{X\left(\frac{j T}{2^{n}}\right)-X\left(\frac{(j-1) T}{2^{n}}\right)}{\sqrt{\sigma^{2} \frac{T}{2^{n}}}}\right)^{2}\right) \\
& =\frac{2 \sigma^{4} T^{2}}{2^{n}}
\end{aligned}
$$

because $\left(\frac{X\left(\frac{j T}{3^{n}}\right)-X\left(\frac{(j-1) T}{2^{n}}\right)}{\sqrt{\sigma^{2} \frac{T}{2^{n}}}}\right)^{2}$ has a chi-s quare distribution with one degree of freedom. (10) implies convergence of $x_{n}$ to $\sigma^{2} T$ in $L_{2}(P)$. To prove, as required, that $Q V \equiv \lim _{n} x_{n}=\sigma^{2} T, P-$ a.e., note that:

$$
\sum_{n=1}^{\infty} E\left(x_{n}-\sigma^{2} T\right)^{2}=2 \sigma^{4} T^{2}<\infty
$$

Since $\left(x_{n}-T\right)^{2} \geq 0, \sum_{n=}^{\infty}$ implies

$$
\begin{aligned}
& \sum_{n=1}^{\infty}\left(x_{n}-\sigma^{2} T\right)^{2}<\infty, P \text {-a.e. } \\
& \lim _{n}\left(x_{n}-\sigma^{2} T\right)^{2}=0, \quad P \text {-a.e. }
\end{aligned}
$$

and

$$
Q V \equiv \lim _{n} x_{n}=\sigma^{2} T \text { P-a.e. }
$$

Proposition 2 allows us to consider (1) with $\sigma=1$.

Formally, let $(\Omega, \mathcal{F}, P)$ be a probability space and $\left\{\mathcal{F}_{t}, t \geq 0\right\}$ a filtration in $(\Omega, \mathcal{F})$. Suppose $\left\{X_{t}\right\}$ is adapted to this filtration and satisfies $(1)$. Let $P_{\theta}^{T}$ be the probability measure generated by $\left\{X_{t}, 0 \leq t \leq T\right\}$ on the space $\left(C[0, T], \mathcal{B}_{T}\right), \mathcal{B}$ corresponding to the Borel sigma-algebra defined in $C[0, T]$. By this we mean:

$$
P_{\theta}^{T}(B)=P\left\{w \in \Omega: X_{t} \in B, \quad B \in \mathcal{B}_{T}\right\}
$$


$P_{\theta}^{T}(B)$ is the measure induced by the process $X_{t}(\theta)$ on $C[0, T]$.

In the same way, let $P_{W}^{T}$ be the probability measure induced by the Wiener process in $C[0, T]$ :

$$
P_{W}^{T}(B)=P\left\{w \in \Omega: W_{t} \in B, \quad B \in \mathcal{B}_{T}\right\}
$$

Then, under regularity conditions ensuring (for all $\theta \in \Theta$ ) the absolute continuity of $P_{\theta}^{T}$ with respect to $P_{W}^{T}$, the Radon-Nikodym derivative $\frac{d P_{\theta}^{T}}{d P_{W}^{T}}$ is given by (see Oksendal (2000), Girsanov's theorem):

$$
\frac{d P_{0}^{T}}{d P_{W}^{T}}=\exp \left\{\int_{0}^{T} h\left(\theta, s, X_{t}\right) d X_{t}-\frac{1}{2} \int_{0}^{T} h^{2}\left(\theta, s, X_{t}\right) d t\right\},[P-a . e]
$$

By definition, the MLE $\hat{\theta}_{T}\left(X_{T}\right)$ of $\theta$ is defined by the measurable map $\hat{\theta}_{T}$ : $\left(\left(C[0, T], \mathcal{B}_{T}\right) \rightarrow(\Theta, \tau)\right.$, such that:

$$
\frac{d P_{\hat{\theta}}^{T}}{d P_{W}^{T}}=\sup _{\theta \in \Theta}\left(\frac{d P_{\theta}^{T}}{d P_{W}^{T}}\right)
$$

where $\tau$ is the $\sigma$-algebra of Borel subsets of $\Theta$.

Example 2 Making $h\left(\theta, s, X_{t}\right)=\theta$, the above equation leads to the maximization of $f(\theta)=\theta X_{T}-\theta^{2} \frac{T}{2}$, with solution $\hat{\theta}=X_{T} / T$.

\subsection{Discretely observed data}

\subsubsection{The case in which the transition densities are known}

There are three well known cases in which the stochastic differential equation (1) is easily solvable, and the corresponding transition functions known: ${ }^{3}$ i) $h\left(\theta, t, X_{t}\right)=\mu X_{t}, \sigma\left(t, X_{t}\right)=\sigma X_{t}$, called geometric Brownian Motion, used, for instance, in the Black and Scholes model; ii) $h\left(\theta, t, X_{t}\right)=\alpha\left(\beta-X_{t}\right), \sigma\left(t, X_{t}\right)=\sigma$, the Orstein-Uhlenbeck process, used, for instance, by Vasicek (1977) to analyze the dynamics of the short-term interest rate and; iii) $h\left(\theta, t, X_{t}\right)=\mu\left(\beta-X_{t}\right)$, $\sigma\left(t, X_{t}\right)=\sigma \sqrt{X_{t}}$, which is the diffusion used in the Cox-Ingersoll-Ross model of the term structure of interest rates. The first of these processes leads to log-normal, the second to normal, and the third to non-central chi-square transition densities.

Example 3 (Maximum likelihood estimation when the transition functions are known): Consider the equation that describes the evolution of the price of the underlying stock in the Black and Scholes model. ${ }^{4}$ This falls into the first case considered above:

$$
d P(t)=\mu P(t) d t+\sigma P(t) d W(t), \quad t \geq 0
$$

\footnotetext{
3Wong (1964) investigates some other particular cases.

${ }^{4}$ Note that this same diffusion equation (usually called geometric Brownian motion) could be used to model different phenomena, in particular populational growth.
} 
Define $Y_{t}=\log \left(P_{t}\right)$. Using Itôś rule:

$$
\left.d Y_{t}=\left(\mu-\frac{1}{2} \sigma^{2}\right) d t+\sigma d W(t)\right)
$$

The above equation implies a normal distribution for the transition densities of $Y_{t}$. Integrating,

$$
\begin{aligned}
\log P_{t} & =\log P_{0}+\left(\mu-\frac{1}{2} \sigma^{2}\right) t+\sigma W(t) \\
P_{t} & =P_{0} e^{\left(\mu-\frac{1}{2} \sigma^{2}\right) t} e^{\sigma W(t)}
\end{aligned}
$$

The conditional distribution of $P_{t}$ given $P_{0}$ is a log normal with mean $\log P_{0}+(\mu-$ $\left.\frac{1}{2} \sigma^{2}\right) t$ and variance $\sigma^{2} t$. The conditional mean of $P_{t}$ given $P_{0}$ can be obtained by using the formula for the moment generating function of a normal random variable of mean $\left(\mu-\frac{1}{2} \sigma^{2}\right)$ and variance $\sigma^{2} t$ :

$$
E\left(P_{t} \mid P_{0}\right)=P_{0} e^{\left(\mu-\frac{1}{2} \sigma^{2}\right) t+\frac{\sigma^{2}}{2} t}=P_{0} e^{\mu t}
$$

Since the transition densities of $Y_{t}$ are known, the application of maximum likelihood in this case follows in a straightforward way. Assuming that the first observation of the Markov process are known, equation (13) leads to the estimators of the mean $(\hat{a})$ and variance $(\hat{b})$ of $\log P_{t}-\log P_{t-1}$ given by:

$$
\begin{aligned}
\hat{a} & =\frac{1}{N} \sum_{t=1}^{N}\left(\log P_{t}-\log P_{t-1}\right) \\
\hat{b} & =\frac{1}{N} \sum_{t=1}^{N}\left(\log P_{t}-\log P_{t-1}-\hat{a}\right)^{2}
\end{aligned}
$$

The Maximum Likelihood Estimators $\mu$ and $\sigma^{2}$ are then given, respectively, by $\hat{a}+\frac{\hat{b}}{2}$ and $\hat{b}$.

One nice feature of continuous modelling is that we can analyze what happens when the time between observations tends to zero. In the present case, this can be done with the help of equation (12). If the time between observations is $h$, $\log P_{t}-\log P_{t-h}$ has a Gaussian distribution with mean $\left(\mu-\frac{\sigma^{2}}{2}\right) h$ and variance $\sigma^{2} h$. The estimators of both $\mu$ and $\sigma^{2}$ (trivially obtained by maximum likelihood), as well as their asymptotic variances, are functions of $h$ and $T$, the number of observations. One can show that the variance of the volatility parameter depends only on the number of observations $T$. It does not depend upon the sampling frequency $h$. The variance of the drift parameter $\mu$, though, depends on both $T$ and $h$. The drift parameter $\mu$ cannot be consistently estimated when the whole time span of the observations is fixed, even if $h \rightarrow 0$ (with $h T \rightarrow k \in \mathbb{R}$ ). ${ }^{5}$

\footnotetext{
${ }^{5}$ The asymptotic variance of the MLE estimator of $\sigma^{2}$ is equal to $2 \sigma^{4} / T$.
} 


\subsubsection{The case in which the transition densities are not known}

As mentioned before, the diffusion for the prices of stocks described in the Black and Scholes model is usually not supported by the data. Here we analyze estimations in more general settings.

Given $n+1$ observations of a diff usion process like (1), consider the data $X(t)$ sampled at non-stochastic dates $t_{0}=0<t_{1}<\ldots<t_{n}$ (equally spaced or not). The joint density of the sample is given by:

$$
p\left(X_{0}, X_{1}, \ldots, X_{n}\right)=p_{0}\left(X_{0}, \theta\right) \Pi_{j=1}^{n} p_{k j}\left(X_{t_{j}}, t_{j} \mid X_{t_{j-1}}, t_{j-1} ; \theta\right)
$$

where $p_{0}\left(X_{0}\right)$ is the marginal density function of $X_{0}$ and $p_{k j}\left(X_{t_{j}}, t_{j} \mid X_{t_{j-1}}\right.$, $\left.t_{j-1} ; \theta\right)$ represents the transition density functions. Such functions are usually not known. In this section, we examine how this problem can be dealt with by using Gaussian distributions to approximate the densities. When the distance between observations is sufficiently small, such approximations lead to reasonable (although biased) estimators.

Sorensen (1995) is the main source of our analysis in this section and the next. In contrast with other methods which use approximate likelihood ratios (Kutoyants, 1984, Yoshida, 1992), the method below uses the exact likelihood of a discretized process. ${ }^{6}$

To see how it works (Prakasa (1999), Florens-Zmirou (1989)), let us start with the diffusion:

$$
d X_{t}=b\left(X_{t}, \theta\right) d t+\sigma\left(X_{t}, \theta\right) d W_{t}, \quad X_{0}=x_{0}
$$

Following Sorensen (1995), we assume that the functions $b\left(X_{t}, \theta\right)$ and $\sigma\left(X_{t}, \theta\right)$ are known, apart from the parameter $\theta$, which varies in a subset $\Theta$ of $\mathbb{R}^{d}$. We discretize this process by assuming that the drift and the diffusion are constant in the time interval ${ }^{7} \Delta_{i}=t_{i}-t_{i-1}$ :

$$
X_{t_{i}}-X_{t_{i-1}}=b\left(X_{t_{i-1}}, \theta\right) \Delta_{i}+\sigma\left(X_{t_{i-1}}, \theta\right)\left(W_{t_{i}}-W_{t_{i-1}}\right)
$$

Since $W_{t_{i}}-W_{t_{i-1}} \mid W_{t_{i-1}} \sim N\left(0, \sigma^{2}\left(X_{t_{i-1}}, \theta\right) \Delta_{i}\right)$, we are actually assuming:

$$
\begin{gathered}
E_{\theta}\left(X_{t_{i}} \mid X_{t_{i-1}}\right)=b\left(X_{t_{i-1}}, \theta\right) \Delta_{i}+X_{t_{i-1}} \\
E_{\theta}\left[\left(X_{t_{i}}-E_{\theta}\left(X_{t_{i}} \mid X_{t_{i-1}}\right)\right)^{2} \mid X_{t_{i-1}}\right]=\sigma^{2}\left(X_{t_{i-1}}, \theta\right) \Delta_{i}
\end{gathered}
$$

${ }^{6}$ Both methods, though, lead to the same estimators, when the diffusion coefficient is constant (see Shoji (1995)).

${ }^{7}$ For equidistant intervals, such approximation, usually called an Euler-Maruyama approximation (Kloeden and Platen, 1992), can be written

$$
X_{t_{i}}-X_{t_{i-1}}=b\left(X_{t_{i-1}}, \theta\right)+\epsilon_{t_{i}}, \epsilon_{t_{i}} \mid X_{t_{i-1}} \sim N\left(0, \sigma^{2}\left(X_{t_{i-1}}, \theta\right)\right)
$$


Note that there are two types of approximation here, one regarding the moments and the other regarding the distribution of the transition densities (as Gaussian). The former usually introduces biases, whereas the latter leads to inefficiency (Sorensen, 2002).

The transition density of the discretized process then reads:

$$
p\left(X_{t_{i}} \mid X_{t_{i-1}}\right)=\frac{1}{\sqrt{2 \pi \sigma^{2}\left(X_{t_{i-1}}, \theta\right) \Delta_{i}}} \exp \left(-\frac{1}{2} \frac{\left(X_{t_{i}}-X_{t_{i-1}}-b\left(X_{t_{i-1}}, \theta\right) \Delta_{i}\right)^{2}}{\sigma^{2}\left(X_{t_{-1}}, \theta\right) \Delta_{i}}\right)
$$

The joint density of $X_{t_{0}} \ldots X_{t_{n}}$ is then given by:

$$
L_{n}(\theta)=\prod_{i=1}^{n} p\left(X_{t_{i}} \mid X_{t_{i-1}}\right) p\left(X_{t_{0}}\right)
$$

Using the last two results and taking logs, the parameters of the problem can be found by the maximization of:

$$
\begin{aligned}
l_{N}(\theta)= & -\frac{1}{2} \sum_{i=1}^{N}\left\{\frac{\left(X_{t_{i}}-X_{t_{i-1}}-b\left(X_{t_{i-1}}, \theta\right) \Delta_{i}\right)^{2}}{\sigma^{2}\left(X_{t_{i-1}}, \theta\right) \Delta_{i}}+\log \left(2 \pi \sigma^{2}\left(X_{t_{i-1}}, \theta\right) \Delta_{i}\right)\right\} \\
& +\log p\left(X_{t_{0}}\right)
\end{aligned}
$$

Taking the derivative with respect to $\theta$ leads to the score function:

$$
\begin{aligned}
& H_{N}(\theta)=\sum_{i=1}^{N} \\
& \quad\left\{\begin{array}{l}
\frac{b_{\theta}\left(X_{t_{i-1}}, \theta\right)}{\sigma^{2}\left(X_{t_{i-1}}, \theta\right)}\left[\left(X_{t_{i}}-X_{t_{i-1}}-b\left(X_{t_{i-1}}, \theta\right) \Delta_{i}\right)\right]+ \\
+\frac{\sigma_{\theta}^{2}\left(X_{t_{i-1}}, \theta\right)}{2\left(\sigma^{2}\left(X_{t_{i-1}}, \theta\right)\right)^{2} \Delta_{i}}\left[\left(X_{t_{i}}-X_{t_{i-1}}-b\left(X_{t_{i-1}}, \theta\right) \Delta_{i}\right)^{2}-\sigma^{2}\left(X_{t_{i-1}}, \theta\right) \Delta_{i}\right]
\end{array}\right\}
\end{aligned}
$$

with the subindex $(.)_{\theta}$ standing for the vector of partial derivatives with respect to $\theta$.

Example 4 The Cox-Ingersoll-and-Ross process which we have first seen in section 2(CIR-SR), satisfies the stochastic differential equation:

$$
d X_{t}=\left(\alpha+\theta X_{t}\right) d t+\sigma \sqrt{X_{t}} d W_{t}
$$

with $\alpha>0, \theta<0$ and $\sigma>0$. Assume that the distance between observation times, $\Delta$, is the same along the sample. As shown by Sorensen (1995), for this process (20) leads to the estimators:

$$
\hat{\alpha}_{n}=\frac{\left(X_{t_{n}}-X_{0}\right)\left(\frac{1}{n} \sum_{i=1}^{n} X_{t_{i-1}}\right)^{-1}-\sum_{i=1}^{n} X_{t_{i-1}}^{-1}\left(X_{t_{i}}-X_{t_{i-1}}\right)}{\Delta\left[n^{2}\left(\sum_{i=1}^{n} X_{t_{i-1}}\right)^{-1}-\sum_{i=}^{n}\right]}
$$




$$
\begin{gathered}
\hat{\theta}_{n}=\frac{\sum_{i=1}^{n} X_{t_{i-1}}^{-1}\left(X_{t_{i}}-X_{t_{i-1}}\right)-\frac{1}{n}\left(X_{t_{n}}-X_{0}\right) \sum_{i=1}^{n} X_{t_{i-1}}^{-1}}{\Delta\left[n-\left(\sum_{i=1}^{n} X_{t_{i-1}}\right)\left(\sum_{i=1}^{n} X_{t_{i-1}}^{-1}\right) / n\right]} \\
\hat{\sigma}_{n}^{2}=\frac{1}{n \Delta} \sum_{i=1}^{n} X_{t_{i-1}}^{-1}\left[X_{t_{i}}-X_{t_{i-1}}-\left(\hat{\alpha}_{n}+\hat{\theta}_{n} X_{t_{i-1}} \Delta\right)\right]^{2}
\end{gathered}
$$

By making, in (20), $\sigma^{2}\left(X_{t_{i-1}}, \theta\right)=v\left(X_{t_{i-1}}, \theta\right)$ we obtain equation (2.3) derived in Sorensen (1995). By deleting the quadratic term (which would be the case when $\sigma^{2}$ is known), we get:

$$
\tilde{H}_{N}(\theta)=\sum_{i=1}^{N}\left\{\frac{b_{\theta}\left(X_{t_{i-1}} ; \theta\right)}{v\left(X_{t_{i-1}} ; \theta\right)}\left[\left(X_{\left(t_{i}\right)}-X_{\left(t_{i-1}\right)}-b\left(X_{t_{i-1}} ; \theta\right) \Delta_{i}\right]\right\}\right.
$$

\section{Consistency and Asymptotic Distribution}

This estimating function has been studied by Dorogovcev (1976), Prakasa $(1983,1988)$, Florens-Zmirou (1989) and Yoshida (1992) in the case when the diff usion coefficient is constant and the parameter $\theta$ is unidimensional. Basically, theses authors have shown that expecting these estimators to be consistent and asymptotically normal requires assuming that the length of the observation interval $\left(n \Delta_{n}\right)$ goes to infinity and that the time between consecutive observations $\left(\Delta_{n}\right)$ goes to zero. Yoshida (1992) proved asymptotic normality imposing $n \Delta_{n}^{2} \rightarrow 0$, whereas Florens-Zmirou (1989) used the less restrictive assumption $n \Delta_{n}^{3} \rightarrow 0$.

Summing up, the estimation by discretization of the transition function works reasonably well when the distance between observation times, $\Delta$, is sufficiently small. Kloeden et al. (1992) confirmed this fact through simulation, whereas Pedersen (1995a) and Bibby and Sorensen (1995) have shown that if $\Delta$ is not small the bias can be severe.

\section{Improving the Approximations for the Moments}

Lemma 1 in Florens-Zmirou $(1989)^{8}$ provides an expansion of $E_{\theta}\left(X_{\Delta} \mid X_{0}=x\right)$ which can be used to improve (16) and (17) to second or higher order. This Lemma will allow us to get better approximations of the average and of the variance of the Gaussian approximations to the transition functions. It reads:

Lemma 3 (Lemma 1 in Florens-Zmirou (1989)): Let $f \in C^{(2 s+2)}$ and denote by $E^{k}$ the conditional expectation w.r.t. $\sigma\left(X_{u}, u \leq k \Delta\right)$ ( the $\sigma$-algebra generated by $\left.\left(X_{u}, u \leq k \Delta\right)\right)$. Then, with $E^{k-1}$ denoting the conditional expectation w.r.t the

\footnotetext{
${ }^{8}$ Florens-Zmirou refers to Dacunha-Castelle and Duflo (1982) as the original reference for the Lemma.
} 
information available at date $(k-1) \Delta$ :

$$
\begin{aligned}
E^{k-1} f\left(X_{k \Delta}\right)= & \sum_{l=0}^{s} \frac{\Delta^{l}}{l !} L^{l} f\left(X_{(k-1) \Delta}\right) \\
& +\int_{0}^{\Delta} \int_{0}^{u_{1}} \ldots \int_{0}^{u_{s}} E^{k-1}\left(L^{s+1} f\right)\left(X_{(k-1) \Delta+\delta}\right) d u_{1} \ldots d u_{s+1}
\end{aligned}
$$

Notice in the expression above the presence of the operator $L$ derived in section 3. This expression, among other things, can be used to determine the bias of the estimator $\vec{\theta}$ derived from (22).

The new equations for the conditional average and variance in (16) and (17) (writing $b$ for $b(x ; \theta)$ and $v$ for $v(x ; \theta)$ ) read:

$$
E_{\theta}\left(X_{\Delta} \mid X_{0}=x\right)=x+\Delta b+\frac{1}{2} \Delta^{2}\left\{b b_{x}+\frac{1}{2} v b_{x x}\right\}+O\left(\Delta^{3}\right)
$$

and

$$
\operatorname{Var}_{\theta}\left(X_{\Delta} \mid X_{0}=x\right)=v \Delta+\Delta^{2}\left[\frac{1}{2} b v_{x}+v\left\{b_{x}+\frac{1}{4} v_{x x}\right\}\right]+O\left(\Delta^{3}\right)
$$

Note that (16) and (17) are a particular case of these expressions, for the cases when $l=1$ in (23).

In order to derive these expressions, note that, from Lemma 1 in FlorensZmirou, making $f(x)=x$, we have::

$$
\begin{aligned}
E_{\theta}\left(X_{\Delta} \mid\right. & \left.X_{0}=x\right)=x+\Delta L x+\frac{\Delta^{2}}{2} L^{2} x+ \\
& +\int_{0}^{\Delta} \int_{0}^{u_{1}} \int_{0}^{u 2} E\left(L^{3} x\right)\left(X_{z}\right) d u_{1} d u_{2} d u_{3}
\end{aligned}
$$

$X_{z}$ standing for $X_{(k-1) \Delta+\delta}$ in Lemma 1.

Since $L x=b(x, \theta), L^{2} x=L b(x, \theta)=b b_{x}+\frac{1}{2} v b_{x x}$ we get (24). The remaining $O\left(\Delta^{3}\right)$ derives from the fact that the (absolute value of the) integrand in (23) is supposed to be bounded (Sorensen (1995), provides sufficient conditions in some particular cases) by some $M \in \mathbb{R}_{+}$and $u_{i} \leq \Delta, i=1,2$, in which case,

$$
\int_{0}^{\Delta} \int_{0}^{u_{1}} \int_{0}^{u 2}\left|E\left(L^{3} x\right)\left(X_{z}\right) d u_{1} d u_{2} d u_{3}\right| \leq M \Delta^{3}
$$

To get (25) we need $E_{\theta}\left(X_{\Delta}^{2} \mid X_{0}=x\right)$. Again, using (24):

$$
\begin{aligned}
E_{\theta}\left(X_{\Delta}^{2} \mid \quad\right. & \left.X_{0}=x\right)=x^{2}+\Delta L x^{2}+\frac{\Delta^{2}}{2} L^{2} x^{2}+ \\
& +\int_{0}^{\Delta} \int_{0}^{u_{1}} \int_{0}^{u 2} E\left(L^{3} x^{2}\right)\left(X_{z}^{2}\right) d u_{1} \ldots d u_{3}
\end{aligned}
$$


We have: $L x^{2}=2 b x+v, L^{2} x^{2}=L(2 b x+v)=b\left(2 b+2 x b_{x}+v_{x}\right)+\frac{1}{2} v\left(2 b_{x}+\right.$ $\left.2 b_{x}+2 x b_{x x}+v_{x x}\right)$. Hence,

$$
\begin{aligned}
E_{\theta}\left(X_{\Delta}^{2} \mid X_{0}=x\right) & =x^{2}+\Delta(2 b x+v)+\frac{\Delta^{2}}{2}\left(b\left(2 b+2 x b_{x}+v_{x}\right)\right. \\
& \left.+\frac{1}{2} v\left(2 b_{x}+2 b_{x}+2 x b_{x x}+v_{x x}\right)\right)
\end{aligned}
$$

From (24) we get:

$$
\left[E_{\theta}\left(X_{\Delta} \mid X_{0}=x\right)\right]^{2}=x^{2}+\Delta 2 b x+\frac{\Delta^{2}}{2}\left(2 b^{2}+2 x b b_{x}+x v b_{x x}\right)+O\left(\Delta^{3}\right)
$$

By subtracting (27) from (26) one gets (25).

Following Sorensen (1995), suppose $\mathrm{X}$ is an ergodic diffusion with invariant probability $\mu_{\theta}$ when $\theta$ is the true parameter. Assuming the process departs from the invariant measure, the expressions (22) and (24) imply a bias of the estimating function (22) given by:

$$
E_{\theta} \tilde{H}_{N}(\theta)=\frac{1}{2} \Delta^{2} n E_{\mu_{\theta}}\left\{b_{x}(\theta)\left[b(\theta) b_{x}(\theta) / v(\theta)+\frac{1}{2} b_{x x}(\theta)\right]\right\}+O\left(n \Delta^{3}\right)
$$

This expression can be obtained by expanding $\tilde{H}_{N}(\theta)$ in (22):

$$
\tilde{H}_{N}\left(\theta_{0}\right)-\bar{H}_{N}(\tilde{\theta})=\tilde{H}_{N}^{\prime}(\tilde{\theta})\left(\theta_{0}-\bar{\theta}\right)
$$

Since $\tilde{H}_{N}(\tilde{\theta})=0$, we have

$$
\left(\theta_{0}-\bar{\theta}\right)=\frac{\tilde{H}_{N}\left(\theta_{0}\right)}{\tilde{H}_{N}^{\prime}(\tilde{\theta})} \rightarrow \frac{\Delta E_{\mu_{0}}\left\{b_{x}(\theta)\left[b(\theta) b_{x}(\theta) / v(\theta)+\frac{1}{2} b_{x x}(\theta)\right]\right\}}{2 E_{\mu_{\theta}}\left\{b_{x}^{2}(\theta) / v(\theta)\right\}}
$$

When the quadratic term in (20) is taken into consideration, the bias (when $\theta$ is the true parameter value) turns out to be:

$$
\begin{aligned}
E_{\theta} \tilde{H}_{N}(\theta) & =\frac{1}{2} \Delta n E_{\mu_{\theta}}\left\{\partial_{\theta} \log v(\theta)\left[\frac{1}{2} b(\theta) \partial_{x} \log v(\theta)+\partial_{x} b(\theta)+\frac{1}{4} \partial_{x}^{2} v(\theta)\right]\right\}+ \\
& +O\left(n \Delta^{2}\right)
\end{aligned}
$$

The important point to notice above is that the bias of the estimating function is of order $\Delta^{2} n$, being therefore considerable even when $\Delta$ is small.

Improving the Estimators by Using Better Approximations for the Moments

Under certain technical conditions, Kessler (1997) devised ways to reduce the bias described above. He retained the idea of approximating the transition densities by a Gaussian distribution, but improved the approximation of the mean and 
of the variance. In order to follow Kessler's approach to the problem we need one definition.

\section{Definition 4 Make}

$$
r_{k}(\Delta, x ; \theta)=\sum_{i=0}^{k} \frac{\Delta^{i}}{i !}
$$

where $f(x)=x$, and where $L_{\theta}^{2}$ denotes the $i^{\text {th }}$ application of the differential operator $L_{\theta}$.

Using the same ideas as the ones detailed in the previous section, but now dealing with expansions of order $k$ (instead of order 2 only), Kessler obtained new approximations for the mean and for the variance of the transition function $\Psi_{k}$ (now, with a remainder term of order $\left.O\left(\Delta^{k+1}\right)\right):^{9}$

$$
\begin{gathered}
E_{\theta}\left(X_{\Delta} \mid X_{0}=x\right)=r_{k}(\Delta, x ; \theta)+O\left(\Delta^{k+1}\right) \\
\Psi_{k}(\Delta, x ; \theta)=\sum_{j=0}^{k} \Delta^{j} \sum_{r=0}^{k-j} \frac{\Delta^{r}}{r !} L_{\theta}^{r} g_{x}^{j},(x)
\end{gathered}
$$

where $g_{x}^{j},(y), j=0,1, \ldots k$ is defined by the expression:

$$
\left(y-r_{k}(\Delta, x ; \theta)\right)^{2}=\sum_{i=0}^{k} \Delta^{j} g_{x}^{j},(y)+O\left(\Delta^{k+1}\right)
$$

As shown in Sorensen (1995), the new approximation of (18) with (29) and (30) replacing (16) and (17) leads to a new approximate score function and to new estimators that perform better than the previous ones.

Example 5 Suppose $X_{t}$ is governed by:

$$
d X_{t}=\theta X_{t} d t+\sigma d W_{t}
$$

Sorensen (1995) has shown that the estimating function based on the approximation detailed in this subsection (with equidistant observations) leads to the estimators (for $k=2$ ):

$$
\begin{gathered}
\tilde{\theta}_{2, n}=\Delta^{-1}\left(\sqrt{2 Q_{n}-1}-1\right) \\
\tilde{\sigma}_{2, n}^{2}=\frac{\frac{1}{n} \sum_{i=1}^{n}\left(X_{t_{i}}-X_{t_{i-1}} Q_{n}\right)}{\Delta+\tilde{\theta}_{2, n} \Delta^{2}+\frac{2}{3} \tilde{\theta}_{2, n}^{2} \Delta^{3}}
\end{gathered}
$$

where

$$
Q_{n}=\frac{\sum_{i=1}^{n} X_{t_{i}} X_{t_{i-1}}}{\sum_{i=1}^{n} X_{t_{i-1}}^{2}}
$$

provided that $Q_{n} \geq 1 / 2$.

\footnotetext{
${ }^{9}$ Remember that in the previous subsection we made an assumption about the boundedness of the integrand.
} 
With these modifications, one gets another score function (which replaces (20)) and other estimators. The estimators so obtained are only slightly biased (Sorensen, 1995), when $\Delta$ is not too large. Under additional conditions, Kessler (1997) shows that the new estimators are consistent and asymptotically normal.

\section{Measuring the Loss of Information Due to Discretization}

Dacunha-Castelle (1986) assumes that the sampling is equidistant and provides a measure of the amount of information lost by discretization in the nonlinear case. The loss is measured, as a function of $\Delta$, in terms of the asymptotic variance of the MLE estimator of the parameters. Such a procedure allows for a determination of how spaced in time the observations can be without leading to a significant problem. The author studies the model (15) [called model $\mathrm{E}^{*}$ ] and also the particular case when $\sigma\left(X_{t}, \theta\right)=\sigma$ (a constant) [called model $\mathrm{E}$ ].

The method expresses the transition density of the Markov chain, $p_{\Delta}$, as a combination of Brownian Bridge functionals. ${ }^{10}$ This is achieved through the use of Girsanov's theorem and Itô's formula. The author concludes that, when $\sigma$ is known, the loss of precision on account of discretization is of order $\Delta^{2}$, whereas when $\sigma$ is unknown the loss is of order $\Delta$.

\section{Martingale Estimating Functions (MEF)}

This section is based on Kessler and Sorensen (1999). We start this section with a Proposition showing that the score function used to derive the MLE in the previous subsections are themselves Martingale estimating functions. The proofs are standard in the literature.

Proposition 5 Under regularity conditions (u.r.c.), the score function is a Martingale.

Proof First we show that the likelihood function is a Martingale and then that the Score Function is a Martingale.

\section{$I$ - The likelihood function is a Martingale:}

Let $P$ and $Q$ be two different probability measures on the space $(\Omega, \mathcal{F})$, and let $\mathcal{F}_{n, n \in \mathbb{N}}$ be a filtration defined in this space. For each $n$, let $P_{n}$ and $Q_{n}$ be the restrictions of $P$ and $Q$ to $\mathcal{F}_{n}$. Suppose $Q_{n}$ is absolutely continuous with respect to $P_{n}$ and make $Z_{n}$ the likelihood function (Radon-Nikodym derivative) $d Q_{n} / d P_{n}$. Then, for sets $A$ in the $\sigma$-algebra $\mathcal{F}_{n-1}$ we have (because the restriction of $P$ and

\footnotetext{
${ }^{10}$ If $\{X(t), t \geq 0\}$ is a Brownian process, a Brownian Bridge is the stochastic process $\{X(t), 0 \leq t \leq 1 \mid X(1)=0\}$. It has mean zero and covariance function $\operatorname{Cov}(X(s), X(t) \mid X(1)=0, s \leq t \leq 1)=s(1-t)$. It can also be represented as $Z(t)=X(t)-t X(1)$ and is very useful in the study of empirical distribution functions.
} 
$Q$ to $\mathcal{F}_{n}$ and the restriction of $P$ and $Q$ to $\mathcal{F}_{n-1}$ must agree on sets in $\left.\mathcal{F}_{n-1}\right)$ :

$$
\int_{A} Z_{n-1} d P=Q(A)=\int_{A} Z_{n} d P
$$

By the definition of conditional expectation, since $A$ is in $\mathcal{F}_{n-1}$ :

$$
\int_{A} E^{\mathcal{F}_{n-1}} Z_{n} d P=\int_{A} Z_{n} d P
$$

Since the probability measure is finite, these equalities imply $E^{\mathcal{F}_{n-1}} Z_{n}=Z_{n-1}$, $P$-a.e.

II - The score function is a Martingale:

Now working with densities defined with respect to the Lebesgue measure, consider the likelihood function $\Lambda_{n}=\exp \left(l_{n}(\theta)-l_{n}\left(\theta_{0}\right)\right)$. Taking the first derivative with respect to theta yields $d \Lambda_{n} / d \theta=\Lambda_{n} d l_{n} / d \theta$. Assuming the derivative can be passed through the integral:

$$
\begin{aligned}
E_{\theta_{0}}^{\mathcal{F}_{n-1}} \Lambda_{n} d l_{n} / d \theta & =E_{\theta_{0}}^{\mathcal{F}_{n-1}} d \Lambda_{n} / d \theta=(d / d \theta) E_{\theta_{0}}^{\mathcal{F}_{n-1}}\left(\Lambda_{n}\right) \\
& =(d / d \theta) \Lambda_{n-1}=\Lambda_{n-1} d l_{n-1} / d \theta
\end{aligned}
$$

The demonstration is concluded by setting $\theta_{0}=\theta$.

We have seen that the use of Gaussian approximations of the transition function leads to biased estimators. We have also seen the biases of such estimators can be somewhat reduced (but not eliminated) by the use of better approximations to the mean and to the variance of the transition density. Such a problem can be avoided by the use of more general MEF. By more general we mean MEF that are not necessarily based on Gaussian approximations to the transition densities of the diffusion processes.

Trying to mimic the score function, such estimating functions $G_{n}(\theta)$ are usually of the form:

$$
G_{n}(\theta)=\sum_{i=1}^{n} g\left(\Delta_{i}, X_{t_{i-1}}, X_{t_{i}} ; \theta\right)
$$

where the functions $g\left(\Delta_{i}, X_{t_{i-1}}, X_{t_{i}} ; \theta\right)$ satisfy:

$$
\int g\left(\Delta_{i}, X_{t_{i-1}}, X_{t_{i}} ; \theta\right) p(\Delta, x, y ; \theta)=0
$$

Here $x$ stands for $X_{t_{i-1}}$ and $y$ for $X_{t_{i}}$. Part of the literature considers functions $g\left(\Delta_{i}, X_{t_{i-1}}, X_{t_{i}} ; \theta\right)$ as polynomials in $y .{ }^{11}$ The approach followed by Kessler and

${ }^{11}$ This was the case, for instance, of the score function (20). However, we have seen that the approximation given by (20) was biased when the time intervals between observations were bounded away from zero. 
Sorensen does not require that the functions $g($.$) are polynomials, even though, in$ some cases, they happen to be. The example below details one such case.

Example 6 Take the Cox-Ingersoll-and-Ross (21) model presented before in this text. As shown by Kessler and Sorensen, for $n=0,1, \ldots$, this model leads to the spectrum:

$$
\Lambda_{\theta}=\{-n \theta\}
$$

with eigenfunctions:

$$
\phi_{i}(X)=\sum_{m=0}^{i}(-1)^{m}\left(\begin{array}{c}
i+2 \alpha \sigma^{-2}-1 \\
i-m
\end{array}\right) \frac{X^{m}}{m !}\left(-2 \theta X \sigma^{-2}\right)
$$

They are based on the eigenfunctions of the generator of the diffusion process. ${ }^{12}$

An important property of an estimating function is being unbiased and being able to identify the correct value of the parameter. Formally, if $\theta_{0}$ stands for the true value of the parameter, one must have:

$$
E_{\theta} G_{n}(\theta)=0 \Leftrightarrow \theta=\theta_{0}
$$

$p(\Delta, x, y ; \theta)$, the transition density from state $\mathrm{x}$ to state $\mathrm{y}$, is usually not known.

Note that (35) means $E_{0}^{\mathcal{F}_{t_{i-1}}} g\left(\Delta_{i}, X_{t_{i-1}}, X_{t_{i}} ; \theta\right)=0$, implying that $G_{n}(\theta)$ is a (difference) martingale and, by the law of iterated expectations, $E_{\theta} G_{n}(\theta)=0$. By an analysis following the same first order-expansion used in (28), equations.(34) and (35) imply that the estimator $\hat{\theta}$ obtained by making $G_{n}(\theta)=0$ is unbiased.

It remains, though, choosing the most adequate $\mathrm{MEF}$ according to some optimizing criterion. In order to do so, consider a class of MEF given by making, in (34):

$$
g\left(\Delta_{i}, X_{t_{i-1}}, X_{t_{i}} ; \theta\right)=\sum_{j=1}^{N} \alpha_{j}(\Delta, x ; \theta) h_{j}\left(\Delta_{i}, X_{t_{i-1}}, X_{t_{i}} ; \theta\right)
$$

Since for each $j$, the event $\left[\alpha_{j}(\Delta, x ; \theta) \in B\right.$, B borelian in $\left.\mathbb{R}^{n}\right] \in \mathcal{F}_{t_{i-1}}$ (where $\left.x=X_{t_{i-1}}\right)$, such functions satisfy (35) if $h_{j}\left(\Delta_{i}, X_{t_{i-1}}, X_{t_{i}} ; \theta\right)$ does. Godambe and Heyde (1987) proposed two possible criteria for the choice of the MEF. The first, called fixed sample criterion, minimizes the distance to the (usually not explicitly known) score function. The second, called asymptotic criterion, chooses the MEF that has the smallest asymptotic variance.

Kessler and Sorensen (1999) provide an analysis of the fixed-sample-criterion type. Under this technique, the estimating function can be viewed as a projection of the score function onto a set of estimating functions of the form (36). Such

${ }^{12}$ To get some intuition linking the eigenfunctions to the estimators of the diffusion process, remember (e.g., Karlin and Taylor (1981)) that the transition density of a diffusion process can be expressed as a series expansion using the eigenfunctions. 
estimating functions are defined by using the eigenfunctions and eigenvalues of the generator $L$ of the underlying diffusion process (which was the object of our analysis in section 3). An important part of their analysis is showing that MEF can be so obtained. This is done in their equation 2.4 , which we present below as a Proposition.

Proposition 6 Consider the diffusion process (8). Let $\phi(x ; \theta)$ be an eigenfunction and $\lambda(\theta)$ an eigenvalue of the operator $L_{\theta}$. Then, under weak regularity conditions (u.r.c.):

$$
E_{\theta}\left[\phi(y ; \theta) \mid X_{t-1}=x\right]=e^{-\lambda(\theta) \Delta} \phi(x ; \theta)
$$

for all $x$ in the state space of $X$ under $P_{\theta}$, implying that

$$
g(y, x ; \theta)=\alpha(x ; \theta)\left\{\phi(y ; \theta)-e^{-\lambda(\theta) \Delta} \phi(x ; \theta)\right\}
$$

is a martingale-difference estimating function.

Proof Make:

$$
Z_{t}=e^{\lambda t} \phi\left(X_{t}\right)
$$

Then, by Itô's formula:

$$
d Z_{t}=e^{\lambda t}\left[\lambda \phi\left(X_{t}\right) d t+\phi \prime\left(X_{t}\right) d X_{t}+\frac{1}{2} \phi \prime \prime\left(X_{t}\right)\left(d X_{t}\right)^{2}\right]
$$

Taking into consideration that $\left(d X_{t}\right)^{2}=\sigma^{2} d t$, and using (8) one gets:

$$
d Z_{t}=e^{\lambda t}\left[\left(\lambda \phi\left(X_{t}\right)+L \phi\left(X_{t}\right) d t+\phi r\left(X_{t}\right) \sigma d W_{t}\right]\right.
$$

Since by assumption $\phi\left(X_{t}\right)$ is an eigenfunction of the operator $L$ with eigenvalue $(-\lambda), L \phi\left(X_{t}\right)+\lambda \phi\left(X_{t}\right)=0$ we have:

$$
d Z_{t}=e^{\lambda t}\left[\phi \prime\left(X_{t}\right) \sigma\left(X_{t}\right) d W_{t}\right]
$$

Integrating this expression,

$$
Z_{t}=Z_{0}+\int_{0}^{t} e^{\lambda_{1} s}\left(\phi \prime\left(X_{s}\right) \sigma\left(X_{s}\right)\right) d W_{s}
$$

Since $\int_{0}^{t} e^{\lambda s}\left(\phi \prime\left(X_{s}\right) \sigma\left(X_{s}\right)\right) d W_{s}$ is a martingale, $Z_{t}$ is a Martingale (for $u<t$, $\left.E^{u} Z_{t}=Z_{u}\right)$. Using this fact in (37) and the definition of $Z_{t}$ one concludes that $E^{t-1} \phi\left(X_{t} ; \theta\right)=e^{-\lambda(\theta) \Delta} \phi\left(X_{t-1} ; \theta\right)=0$, as required.

Kessler and Sorensen show that the estimators so obtained are, u.r.c., consistent and asymptotically normal (by using the Martingale Central Limit Theorem, (Billingsley, 1961)).

The consistency and asymptotic normality of the estimators derived by Kessler and Sorensen do not require the assumption, as the analysis in section 4 did, that the time between observations tends to zero. This is an important advantage of such estimators, since $\Delta \rightarrow 0$ is usually not observed by real data. 


\section{GMM Estimation}

As detailed in the seminal paper by Hansen (1982), a GMM estimator is obtained by minimizing a criterion function of sample moments which are derived from orthogonality conditions. Hansen and Scheinkman (1995) show how to generate moment conditions for continuous-time Markov processes with discrete-time sampling. The basic idea pursued by the authors is that such processes can be characterized by means of forward or backward infinitesimal generators (see section 3). Also, when the processes are stationary these generators can be employed to derive moment conditions that can be used for estimation purposes by the application of Hansen's (1982) GMM.

Note that, by the law of iterated expectations:

$$
\int_{R^{n}} f d \mathcal{Q}=\int_{R^{n}} \Gamma_{t} f d \mathcal{Q}
$$

Using the framework developed in section 3 , since $\frac{1}{t}\left(\Gamma_{t} f-f\right)$ converges in $\mathrm{L}^{2}(\mathcal{Q})$ to $\Lambda_{t} f,(38)$ implies:

$$
\int_{R^{n}} \Lambda f d \mathcal{Q}=\lim _{t \rightarrow 0} \frac{1}{t} \int_{R^{n}}\left(\Gamma_{t} f-f\right) d \mathcal{Q}=0
$$

and the (first set of) moments conditions ${ }^{13}$ :

$$
E\left[\Lambda f\left(X_{t}\right)\right]=0 \text { for all } f \in \Psi
$$

This is a well-known link between the generator and the stationary distribution. A second set of moment conditions is derived by the authors using the reverse-time process:

$$
E\left[\Lambda f\left(X_{t+1}\right) \check{f}\left(X_{t}\right)-f\left(X_{t+1}\right) \check{\Lambda} \dot{f}\left(X_{t}\right)\right]=0 \text { for all } f \in \Psi, \dot{f} \in \dot{\Psi}
$$

where $\check{f}$ and $\dot{\Psi}$ are defined as in section 3 , but now with respect to the reverse process. Note that only the second set of moments, by depending on the variables measures in two consecutive points of time, directly captures the Markovian features of the model.

In practice, GMM estimation usually starts with the employment of an EulerMaruyama discretization. Using the notation here developed, take, for instance, the diffusion process:

$$
d X_{t}=g\left(X_{t}\right) d t+\sigma d W_{t}
$$

By using the Eulyer-Maruyama approximation:

$$
X_{t}-X_{t-1}=g\left(X_{t_{i-1}}\right)+\sigma\left(X_{t_{i}}-X_{t_{i-1}}\right)
$$

\footnotetext{
${ }^{13}$ Remember the definition of $\Psi$ from section 3 .
} 
Next, define $e_{t_{i}}=X_{t_{i}}-X_{t_{i-1}}-g\left(X_{t_{i-1}}\right), f_{t_{i}}=\left(X_{t_{i}}-X_{t_{i-1}}-g\left(X_{t_{i-1}}\right)\right)^{2}-\sigma^{2} \Delta t$ and $z_{t}=\left(e_{t_{i}}, f_{t_{i}}, X_{t_{i-1}} e_{t_{i}}, X_{t_{i-1}} f_{t_{i}}\right)$. Under the null, $E_{t-1} z_{t_{i}}=0$. Replace this conditional expectation by its sample counterpart to obtain a quadratic form, the maximization of which leads to the parameter estimates (see the second example below).

Example 7 Consider the diffusion process:

$$
d p=-a(p-\mu) d t+\sigma d W_{t}, p(0)=p_{0}>0, a>0
$$

Campbell et al. (1997) present a heuristic development of this example. The direct use of the results we proved in section 3, though, allows for a direct formal approach. Taking $f$ as the identity function and applying the generator $\Lambda$ to (41):

$$
\Lambda(d p)=-a(p-\mu)
$$

Using (39) leads to the first moment condition:

$$
E p=\mu
$$

Now, instead of taking $f=I$ (Identity) in the above procedure, take a generic test function $f$ (for instance, $f()=.(.)^{n}, n \in N$ ). Make $Y_{t}=f\left(p_{t}\right)$ and apply Itô's Lemma:

$$
d f\left(p_{t}\right)=d Y_{t}=\left[-f^{\prime}(p) a(p-\mu)+\frac{1}{2} f^{\prime \prime}(p) \sigma^{2}\right] d t+f^{\prime}(p) o \cdot d W
$$

Using (39) once more:

$$
E\left[-f^{\prime}(p) a(p-\mu)+\frac{1}{2} f^{\prime \prime}(p) \sigma^{2}\right]=0
$$

Using (40):

$$
\begin{gathered}
E\left\{\left[-a(p-\mu) \phi^{\prime}\left(X_{t+1}\right)+\frac{1}{2} o^{2} \phi^{\prime \prime}\left(X_{t}\right)\right] \check{\phi}\left(X_{t}\right)-\phi\left(X_{t+1}\right)\right. \\
{\left[-a(p-\mu) \check{\phi}^{\prime}\left(X_{t}\right)+\frac{1}{2} o^{2} \check{\phi}^{\prime \prime}\left(X_{t}\right]\right\}=0}
\end{gathered}
$$

Equations (39) and (40) define an infinite number of moment conditions, depending on the choice of $f$. Under the regularity conditions provided by the authors, $G M M$ can then be applied. 
Example 8 As a second example, and also for the purpose of comparison with one of the other estimation procedures we have seen in this paper, let's go back to the CIR-SR model of interest rates presented in section 2 and in example 4. Chan et al. (1992) estimate this process using GMM. Denoting by $X_{t}$ the interest rate at time $t$, these authors estimate a discrete time version of the CIR-SR model given by:

$$
X_{t+1}-X_{t}=\alpha+\theta X_{t}+\epsilon_{t+1}
$$

in which $E \epsilon_{t+1}=0$ and $E\left(\epsilon_{t+1}\right)^{2}=\sigma^{2} X_{t}$. The estimation goes as follows. Make $\beta=\left(\alpha, \theta, \sigma^{2}\right)$. Next, define:

$$
f_{t}(\beta)=\left[\begin{array}{l}
\epsilon_{t+1} \\
\epsilon_{t+1} X_{t} \\
\epsilon_{t+1}^{2}-\sigma^{2} X_{t} \\
\left(\epsilon_{t+1}^{2}-\sigma^{2} X_{t}\right) X_{t}
\end{array}\right]
$$

Under the null, $E f_{t}(\beta)=0$. The estimators associated with the GMM method are then found simply by replacing $E f_{t}(\beta)$ with its sample counterpart. The process leads to a minimization of a quadratic form.

Example 9 (Brazilian Financial Time Series) For an estimation of the CIR-SR model (and other interest-rate models as well) with Brazilian financial time series, the reader can refer to Barrossi-Filho and Dario (2003). In another section of the paper, these authors also use Monte Carlo simulation methods to compare finite-sample distribution properties of the GMM and of the Euler-Maruyama approximation.

\section{Comparing Different Estimators}

Comparisons of the estimators studied in this (partial) survey are relatively scarce in the literature. Changes of the time interval used for the simulations is one reason for this. The unavoidable aliasing phenomenon (the fact that distinct continuous-time processes may look identical when sampled at discrete points of time), can lead to different results, making it extremely difficult to make general statements about relative efficiencies of one or another estimator.

Checking the influence of changes in discrete-time sampling interval requires repeating the experiments several times (once the number of sample paths has been set, depending upon the discrete time intervals, the number of sample points will, of course, vary accordingly).

There is still the problem that, when the diffusion term is state dependent, it is possible that the numerical values of the sample path diverges, because the variance becomes large. This usually implies the necessity of a transformation of variables (in order to obtain a stochastic differential equation with a constant diffusion coefficient), and of a reversal of the transformation later. 
Shoji and Ozaki (1997) is one of the few examples performing such comparisons. This paper develops Monte Carlo experiments using five different methods of obtaining MLE estimators (including the Euler-Maruyama approach and GMM, both of which we have reviewed here). Two different diffusion processes are considered, the first with linear drift and state-dependent diffusion coefficients and the other with nonlinear drift and constant diffusion coefficients:

$$
d X_{t}=\left(\alpha+\beta X_{t}\right) d t+\sigma X_{t} d W_{t}
$$

and

$$
d X_{t}=\alpha X_{t}^{3} d t+\sigma d W
$$

In the experiments, the number of sample paths is fixed and the number of sample points varies depending upon the discrete time interval. Note, in (45), that the diffusion term is state dependent, thereby causing the type of problem we have mentioned above in this section, requiring a change of variable.

After making Monte Carlo simulations, these authors concluded, regarding (45), that the GMM performed somewhat inferior to the Euler-Maruyama approach (a poorer performance of GMM estimators is also found by Barrossi-Filho and Dario (2003)). The GMM, though, did a little better in (46) than in (45).

The interested reader can refer to Jiang and Knight (1999) and Ait-Sahalia and Mykland (2000) for further study of the comparison among different estimators. Jiang and Knight (1999) use Monte Carlo simulation to investigate the finite-sample properties of various estimators, including GMM estimators and some others which we have not reviewed here. Ait-Sahalia and Mykland (2000) provide a general method to compare the performance of a variety of estimators of diffusion processes, when the data are not only discretely sampled in time but, in addition, the time separating successive observations may possibly be random. GMM and the Euler-Maruyama approximation are among the methods assessed by these authors.

\section{Conclusion}

In this paper, we have provided a partial review of the literature regarding the statistical estimation of diffusion processes by investigating three of the available estimation methods: an improvement of the Euler-Maruyama discretization scheme, the employment of Martingale Estimating Functions and the application of Generalized Method of Moments (GMM).

In order to interest the reader in this important area of statistical research, we have provided several examples, a final section with a brief review of a comparison between the performance of two of the methods that we have investigated, as well as detailed formalizations of some of the analytical developments made in the original texts. This is an area of research that is presently very active and of 
particular interest to economists, particularly, at the present stage, because of its several applications in finance and macroeconomics.

A research parallel to this one involves studying the statistical consequences of the random sampling (as opposed to the effects on which we have concentrated here, of the discrete sampling) of the diffusion processes. Ait-Sahalia and Mykland (2003) are a seminal reference in this area, where a new body of research is expected to emerge.

\section{References}

Ait-Sahalia, Y. (2002). Maximum likelihood estimation of discretely sampled diffusions: A closed-form approximation approach. Econometrica, 70(1):223-262.

Ait-Sahalia, Y. \& Mykland, P. (2000). A comparison of estimators of continuoustime diffusions. Annals of the World Congress of the Econometric Society, Seattle, 2000.

Ait-Sahalia, Y. \& Mykland, P. (2003). The effects of random and discrete sampling when estimating continuous-time diffusions. Econometrica, 71(2):483-549.

Barrossi-Filho, M. \& Dario, A. G. (2003). Estimaçāo de modelos em tempo contínuo: Taxa de juros de curto prazo e Simulações de Monte Carlo. Mimeo, FEA/USP.

Basawa, I. V. \& Prakasa, R., B. L. S. (1980). Statistical inference for stochastic processes. Academic Press, London.

Bibby, B. M. \& Sorensen, M. (1995). Martingale estimation functions for discretely observed diffusion processes. Bernoulli, 1:17-39.

Billingsley, P. (1961). The Lindberg-Levy theorem for Martingales. Proceedings of the American Mathematical Society, 12:788-792.

Black, F. \& Scholes, M. (1973). The pricing of options and corporate liabilities. Journal of Political Economy, 81(3):637-654.

Campbell, J. Y., Lo, A. W., \& MacKinlay, A. C. (1997). The econometrics of financial markets. Princeton University Press.

Chan, K., Karolyi, G., Longstaff, F., \& Sanders, A. (1992). An empirical comparison of alternative models of short-term interest rate. Journal of Finance, XLVII:1209-1227.

Cox, J., Ingersoll, J., \& Ross, S. (1985). A theory of the term structure of interest rates. Econometrica, 53:385-408. 
Dacunha-Castelle, D. (1986). Estimation of the coefficients of a diffusion from discrete observations. Stochastics, 19:263-284.

Dacunha-Castelle, D. \& Duflo, M. (1982). Probability and statistics. Springer Verlag, 2. New York.

Dorogovcev, A. J. (1976). The consistency of an estimate of a parameter of a stochastic differential equation. Theory of Probability and Mathematical Statistics, 10:73-82.

Florens-Zmirou, D. (1989). Approximate discrete-time schemes for statistics of diffusion processes. Statistics, 20:547-557.

Godambe, V. P. \& Heyde, C. (1987). Quasi likelihood and optimal estimation. International Statististics Review, 55:231-244.

Gourieroux, C. \& Jasiak, J. (2001). Financial econometrics: Problems, models, and methods. Princeton Series in Finance.

Hansen, L. P. (1982). Large sample properties of generalized method of moments estimators. Econometrica, 50:1029-1054.

Hansen, L. P. \& Scheinkman, J. A. (1995). Back to the future: Generating moment implications for continuous-time Markov processes. Econometrica, 63(4):767804.

Jiang, G. \& Knight, J. L. (1999). Finite sample comparison of alternative estimators of Itô diffusion processes: A Monte Carlo study. Journal of Computational Finance, 2(30).

Karatzas, I. \& Shreve, S. E. (1991). Brownian motion and stochastic calculus. Second Edition, Spring Verlag.

Karlin, S. \& Taylor, H. M. (1981). A second course in stochastic process. Orlando: Academic Press.

Kessler, M. (1997). Estimation of an ergodic diffusion from discrete observations. Scandinavian Journal of Statistics, 24(2):211.

Kessler, M. \& Sorensen, M. (1999). Estimating equations based on Eigenfunctions for a discretely observed diffusion process. Bernoulli, 5(2):299-314.

Kloeden, P. E. \& Platen, E. (1992). Numerical solution of stochastic differential equations. Springer-Verlag, New York.

Kloeden, P. E., Platen, E., Schurz, H., \& Sorensen, M. (1992). On the effects of discretization on estimators of drift parameters for diffusion processes. Research Report, 249. Department of Theoretical Statistics, University of Aarhus. 
Krylov, N. Z. (1980). Controlled diffusion processes. Springer Verlag.

Kutoyants, Y. A. (1984). Parameter estimation for stochastic processes. (translated and edited by B. L. S. Prakasa Rao). Berlin, Heldmann.

Oksendal, B. (2000). Stochastic differential equations. 5th Edition, Springer Verlag.

Pedersen, A. R. (1995a). Consistency and asymptotic normality of an approximate maximum likelihood estimator for discretely observed diffusion processes. Bernoulli, 1:257-279.

Pedersen, A. R. (1995b). A new approach to maximum likelihood estimation for stochastic differential equations based on discrete observations. Scandinavian Journal of Statististics, 22:55-71.

Phillips, A. W. (1959). The estimation of parameters in systems of stochastic differential equations. Biometrika, 46:67-76.

Prakasa, R. B. L. S. (1983). Asymptotic theory of non-linear least squares estimator for diffusion processes. Math. Operationsforsch. Statist.

Prakasa, R. B. L. S. (1988). Statistical inference from sampled data for stochastic processes. Contemporary Mathematics, 80:249-284.

Prakasa, R. B. L. S. (1999). Statistical inference for diffusion-type processes. Kendall's Library of Statistics, 8.

Shoji, I. (1995). Consistency of the estimator derived from the Euler method. Research Memo, 587. Institute of Statistical Mathematics, Tokyo.

Shoji, I. \& Ozaki, T. (1997). Comparative study of estimation methods for continuous time stochastic processes. Journal of Time Series Analysis, 18(5).

Sorensen, H. (2002). Parametric inference for diffusion processes observed at discrete points in time: A survey. Mimeo, University of Copenhagen.

Sorensen, M. (1995). Estimating functions for discretely observed diffusions: A review.

Vasicek, O. (1977). An equilibrium characterization of the term structure of interest rates. Journal of Financial Economics, 5:177-188.

Wong, E. (1964). The construction of a class of stationary Markov processes. In Bellman, R., editor, Mathematical Physics and Engineering. Proceedings of Simposia in Applied Mathematics. Stochastic Processes in Mathematical Physics and Engineering, chapter 16, pages 264-276. American Mathematical Society, Providence, RI. 
Yoshida, N. (1992). Estimation for diffusion processes from discrete observations. Journal of Multivariate Analysis, 41:220-242. 



\section{ACKNOWLEDGEMENT}

The Editor of The Brazilian Review of Econometrics would like to thank the following referees for their contribution during the 2003-2004 period.

$\begin{array}{ll}\text { Eduardo Andrade } & \text { (Ibmec-SP) } \\ \text { Eurilton Araujo } & \text { (Ibmec-SP) } \\ \text { Juliano Assunção } & \text { (PUC-RIO) } \\ \text { Renato M. Assunção } & \text { (ICEx/UFMG) } \\ \text { Tara Baidya } & \text { (PUC-RIO) } \\ \text { Flávio Ataliba Barreto } & \text { (CAEN-UFC) } \\ \text { Walter Belluzzo } & \text { (FEA-USP) } \\ \text { Ney Roberto Ottoni de Brito } & \text { (Ney O. Brito \& Associados e UFRJ) } \\ \text { Rodrigo de Losso S. Bueno } & \text { (USP \& University of Chicago) } \\ \text { Mauricio Bugarin } & \text { (UnB) } \\ \text { Mirta Bugarin } & \text { (UnB) } \\ \text { Antonio Gledson de Carvalho } & \text { (FEA-USP) } \\ \text { Eui Jung Chang } & \text { (Banco Central do Brasil) } \\ \text { Newton C. Da Costa } & \text { (UFSC) } \\ \text { Oswaldo Luiz V. Costa } & \text { (Escola Politécnica da USP) } \\ \text { Samir Cury } & \text { (EAESP-FGV) } \\ \text { Rubens Penha Cysne } & \text { (EPGE-FGV) } \\ \text { William Eid } & \text { (EAESP-FGV) } \\ \text { Roberto Ellery } & \text { (UnB) } \\ \text { Luís Façanha } & \text { (IE/UFRJ) } \\ \text { José Fajardo } & \text { (Ibmec-RJ) } \\ \text { Aquiles Farias } & \text { (UnB) } \\ \text { Cristiano Fernandes } & \text { (PUC-Rio) } \\ \text { Reynaldo Fernandes } & \text { (FEA-USP) } \\ \text { Gerson Francisco } & \text { (Instituto de Física Teórica, UNESP) } \\ \text { Paulo Springer de Freitas } & \text { (Banco Central do Brasil) } \\ \text { Ana Beatriz Galvão } & \text { (Ibmec-SP) } \\ \text { Carlos Eduardo S. Gonçalves } & \text { (FEA-USP) } \\ \text { Solange Maria Guerra } & \text { (Banco Central do Brasil) } \\ \text { Osmani Guillén } & \text { (EPGE-FGV \& Banco Central do Brasil) } \\ \text { Rodolfo Hoffmann } & \text { (IE-UNICAMP) } \\ \text { João Victor Issler } & \text { (EPGE-FGV) } \\ \text { Fabio Kanczuk } & \text { (FEA-USP) } \\ \text { Sergio Kannebley } & \text { (FEA-USP) } \\ \text { Marcia Saraiva Leon } & \text { (Banco Central do Brasil) } \\ \text { Jorge C. Lima } & \text { (BNDES) } \\ & \\ & \end{array}$





\section{NOTE \\ ADRIANO ROMARIZ DUARTE AWARD}

In 1994 the Brazilian Econometric Society instituted the Adriano Romariz Duarte Award. Since then, the award has been given every even year to the best article published in the Brazilian Review of Econometrics. The next award will be given to the articles published in volumes 23 and 24 .

According to the rules governing the Award, the Editor will appoint an award commission which will select the winnning article.

A list of previous award winners is:

\section{4}

"Strategic Behavior in Ascending-Price Multiple-Object Auctions", by Flávio Marques Menezes.

\section{6}

"Déficit Público, A Sustentabilidade do Crescimento das Dívidas Interna e Externa, Senhoriagem e Inflação: Uma Análise do Regime Monetário Brasileiro", by Affonso Celso Pastore.

"Mecanismos de Admissão de Candidatos à Instituiçōes. Modelagem e Análise à Luz da Teoria dos Jogos", by Marilda Sotomayor.

1998

"Estimadores Corrigidos para Modelos SUR Não-Lineares", by Gauss M. Cordeiro and Klaus L.P. de Vasconcellos.

"Cournotian Competition under Knigthian Uncertainty", by Sérgio Ribeiro da Costa Werlang and Hugo Pedro Boff.

\section{0}

"The Slippery Slope: Explaining the Increase in Extreme Poverty in Urban Brazil, 1976 - 1996", by Francisco H. G. Ferreira and Ricardo Paes de Barros.

\section{2}

"Towards a Truthful Land Taxation Mechanism in Brazil", by Humberto Luiz Ataide Moreira and Juliano Junqueira Assunção.

Honorable Mention

"A Monthly Indicator of Brazilian GDP", by Marcelle Chauvet. 



\section{Subscription Form}

To become a subscriber to The Brazilian Review of Econometrics you should fill out this form and send it to: The Brazilian Review of Econometrics -

Name

A/C: Angela Maria Bevilacqua

Praia de Botafogo, 190 - 10o. andar sala 1032

222250-900 Botafogo, Rio de Janeiro, RJ., Brasil

Tel.(5521) 2551-4658

Fax (5521)2552-4898

sbe@fgv.br - http: //www.sbe.org.br

Subscriptions

US\$ 100 / R\$150,00

Institutions and new subscribers

US\$ 50 / R $\$ 75,00$

Renewals for individual subscribers

US\$ 25 / R \$37,50 Students

Institution

Address

City

State

Postal Code

Country

Telephone

Fax

E-mail

Year of Subscription

Amount in US\$ or R\$ 


\section{Methods of Payment}

\section{Checks}

Please send a check with the value of your subscription payable to Sociedade Brasileira de Econometria which can be drawn in a Brazilian bank to the SBE office.

After we receive your check we will send you a receipt, as well as the issues of the Review that correspond to your subscription.

Checks and subscription form should be sent to:

SBE The Brazilian Review of Econometrics

A/C Angela Maria Bevilacqua

Praia de Botafogo, 190 - $10^{\circ}$ Andar - Sala 1032

22250-900 - Botafogo - Rio de Janeiro, RJ., Brasil

Tel. (5521) 2551-4658 - Fax (5521) 2552-4898

sbe@fgv.br - http://www.sbe.org.br/

\section{Bank Deposit}

A bank deposit must be made in the society's account at Banco do Brasil.

Please send us a fax (5521) 25524898 with a copy of your deposit slip attached to your subscription form. The fax is your proof of subscription.

Account Information

Banco do Brasil - Rio de Janeiro - RJ Brasil

Agency - Botafogo

Number 0287-9

Account 112.842-6 


\section{Payment with a Credit Card}

Credit card Number

Expiration Date

Amount

Name of the Card

Signature

Date

We accept Diners, Master Card and Credicard.

Send subscription form to:

SBE The Brazilian Review of Econometrics

A/C: Angela Maria Bevilacqua

Praia de Botafogo, 190 - 10o. andar - sala 1032

22250-900 Botafogo, Rio de Janeiro, RJ., Brasil

Tel. (5521) 2551-4658

Fax (5521) 2552-4898

sbe@fgv.br - http: //www.sbe.org.br 



\section{Submission of Manuscripts to the Brazilian Review of Econometrics}

1. Four copies of the original manuscript should be sent to:

Angela Maria Bevilacqua

Editorial Assistant, Brazilian Review of Econometrics

EPGE - Fundação Getulio Vargas

Praia de Botafogo, 190 - 10o. Andar

Rio de Janeiro, RJ 22250-900 Brazil.

Two copies of the manuscript should not have the author's name indicated or revealed in any manner. The manuscripts must be accompanied by a submission letter and be written in English or Portuguese. Submission of a paper is held to imply its contents represent original and unpublished work, and that it has not been submitted elsewhere. Manuscripts are always refereed and may be rejected, returned for specified revisions or accepted.

2. Manuscripts should have an opening page containing the title, the author's name(s), address(es), e-mail and affiliation. It should also contain a set of Key Words and Phrases, a primary and secondary Journal of Economic Literature classification for the manuscript and an Abstract in English of at most 150 words.

3. Manuscripts should be typed with double line spacing on standard size paper, preferably $8.5 \times 11$ inches, in a font size of 10 or $12 \mathrm{cpi}$. The typing area of all pages should be no more than $5.5 \times 8.5$ inches $(14 \times 21.6 \mathrm{~cm})$.

4. Tables should be typed as part of the text, but in such a way as to avoid confusion with text, extra space should be left above and below the table. Tables, including title, legend, label or number, should fit within the standard typing area and should not run over to the next page.

5. Graphs and other numbered figures should be separate from the text, sized to fit within the width and height of the typed pages and be ready for printing.

6. References should be indicated in the text by the author's name and the year of publication in parentheses. All references should then be collected in alphabetical order at the end of the manuscript.

7. Any acknowledgements, including citations to supporting grants and contracts, should be typed as text and placed before the references at the end of the manuscript. 
